\title{
LA FORMACIÓN SOCIAL DE FRONTERAS DE LA PUNA Y EL ALTIPLANO BREVE ESTUDIO GEO-HISTÓRICO SOBRE EL NORTE ARGENTINO Y EL SUR POTOSINO
}

\section{Sergio Iván BRATICEVIC ${ }^{1}$}

Pablo CRUZ ${ }^{2}$

\section{Resumen}

El empleo de la categoría formación social de fronteras se enmarca en un análisis geohistórico global, con el objetivo de caracterizar territorios heterogéneos de alta complejidad social. Para el caso específico, se realiza un análisis sobre las interacciones entre espacios pertenecientes a distintos estados nacionales, en función de sus especificidades estatales y sus continuidades productivas. El desarrollo reciente en materia de infraestructura -junto con la estabilidad económica en Bolivia- propiciaron el mejoramiento general de las condiciones de vida. En contraposición, Argentina reforzó los dispositivos de "seguridad" sobre la frontera, mientras que no se realizaron grandes inversiones de capital a escala regional. Todo lo descripto activó ciertas discontinuidades en los circuitos económicos típicos de la frontera que se analizan en este artículo.

Palabras clave: formación social; frontera; espacio; Altiplano, Puna.

\section{Summary}

The example of social frontier formation is immersed in a geo-historical analysis which aim is to characterize heterogeneous territories with a vast social complexity. In this particular case, we have performed an exploration upon the national estate interactions;

Fecha de recepción del artículo Octubre 2019

Fecha de evaluación: Mayo 2020

\footnotetext{
${ }^{1}$ Geógrafo y Doctor en Antropología. Investigador Asistente en UE-CISOR, CONICET-UNJu. Correo electrónico: sergiobraticevic@gmail.com

${ }^{2}$ Doctor en Antropología y Arqueología. Investigador Independiente en UE-CISOR, CONICET-UNJu. Correo electrónico: pablocruz@,conicet.gov.ar
} 
regarding their own estate particularities and their productive similarities. The recent development in infrastructure- along with economic stability in Bolivia- encouraged the social welfare. In contrast to Bolivian improvements, Argentina enforced "safety" devices along the border meanwhile the lack of a regional investment plan became notorious. According to this overall picture this article aim is to analyze the discontinuities of typical economic border circuits.

Key words: social formation; border; space; Altiplano; Puna.

\section{Résumé}

L'idée de formation sociale de frontière est fondée sur une analyse géo-historique lequel essai de caractériser des territoires que partage une grande hétérogénéité de complexité sociale. En ce cas particulier, on a conduit une analyse sur les interactions entre les pays attenants et ces spécificités états et ces productions partages. Le récent développement en matière d'infrastructure et la stabilité économique bolivienne à favorise une meilleure condition de vie. Par opposition, l'Argentine a fait des grandes avancées en matière de "sécurité " limitrophe sans grands investissement dans la région. Sur tous ce qu'on a décrit ici c'est que les circuits économiques typiques de frontière ont été touchés et sont-ils analyses en cet article

Mots clés : formation sociale ; frontière ; espace ; Altiplano ; Puna.

\section{Presentación y conceptualización}

La noción formación social de fronteras proviene de una reconocida contribución realizada por Marx en la Introducción general a la crítica de la economía política (1857). Afirmaba, que la ley económica fundamental de las formaciones económico-sociales está regida por la ley fundamental del modo de producción dominante. Asimismo, esta norma rectora -la producción de plusvalor- es la finalidad directa y móvil determinante de la producción. Según este criterio, en cualquier forma de sociedad existe un modo de producción dominante que impone sus reglas sobre las restantes formas económicas pretéritas o arcaicas, modificando así sus particularidades y características.

Es aquí donde se puede reconocer la génesis de la re-conceptualización de los modos de producción y su articulación: la formación económico-social. La tradición académica 
marxista francesa contiene el desarrollo más acabado de esta categoría, durante la década de 1970, a partir del texto Para leer El Capital, de Althusser y Balibar (1974). La aplicación de este marco conceptual ha sido criticada desde la antropología debido a su sesgo funcionalista y positivista, dado que las sociedades precapitalistas son analizadas como una totalidad social dentro de la formación económica actual o presente. Además, no identificaba las transformaciones sufridas a raíz del avance del frente agrario capitalista.

Pareciera, entonces, que se tratara de una mera coincidencia espacial y que la superposición de modos de producción con el tiempo iría dando lugar a la forma de organización dominante. Se pierden, así, las particularidades que no obedecen a las normas de acumulación típicamente capitalistas, como se describe con los procesos de subsunción. Por su parte, estas interpretaciones, un tanto estáticas y generalizantes de la articulación de los modos de producción, responden a una visión evolucionista, propia del siglo XIX, y a su relectura estructuralista durante la década de 1970 (Trinchero, 2000).

Ahora bien, si se revisa esta perspectiva, se podría hablar de refuncionalización de relaciones de producción preexistentes orientadas a las reglas de la reproducción ampliada de capital, manteniendo formas domésticas de reproducción no necesariamente capitalistas (subsunción del trabajo al capital). En efecto, se trata de los casos en los cuales el sector doméstico ha mantenido la propiedad de los medios de producción, cediendo el control de las condiciones de existencia, ya que el capital ha introducido un conjunto de criterios propios sobre la producción doméstica (a través de nuevos patrones de intercambio y fijación de precios de los productos campesinos).

\section{Formación social de fronteras}

Sintéticamente, se puede afirmar que la categoría formación social de fronteras expresa particularidad y totalidad de esferas productivas diversas, dentro de un espacio sin límites precisos o estáticos, a partir de la siguiente idea: distintas relaciones de producción que se estructuran de acuerdo a su funcionalidad con las normas de reproducción sistémica, y no como distintos modos de producción articulados entre sí. Diferentes autores han trabajado esta noción, especialmente desde la antropología económica (Gordillo, 1992 y 
1995; Trinchero, Piccinini y Gordillo, 1992; Trinchero, 2000 y 2007; Trinchero, Belli y Slavutsky, 2004).

Resultan fundamentales los aportes de Trinchero (2000), quien desarrolla un análisis histórico del Chaco central argentino proponiendo esta categoría analítica. Su objetivo es caracterizar territorios heterogéneos de alta complejidad social, en donde se despliegan particulares relaciones de producción capitalistas expresadas en la conjunción de situaciones de construcción de fronteras políticas, productivas y culturales. Es así como quedan reflejadas las dinámicas del capitalismo en la formación social de fronteras que representa el Chaco, desde una historización completa que abre camino a los estudios de las fronteras y los frentes agropecuarios en áreas marginales del Estado-Nación argentino.

Figura 1. Composición de fronteras en la formación social.

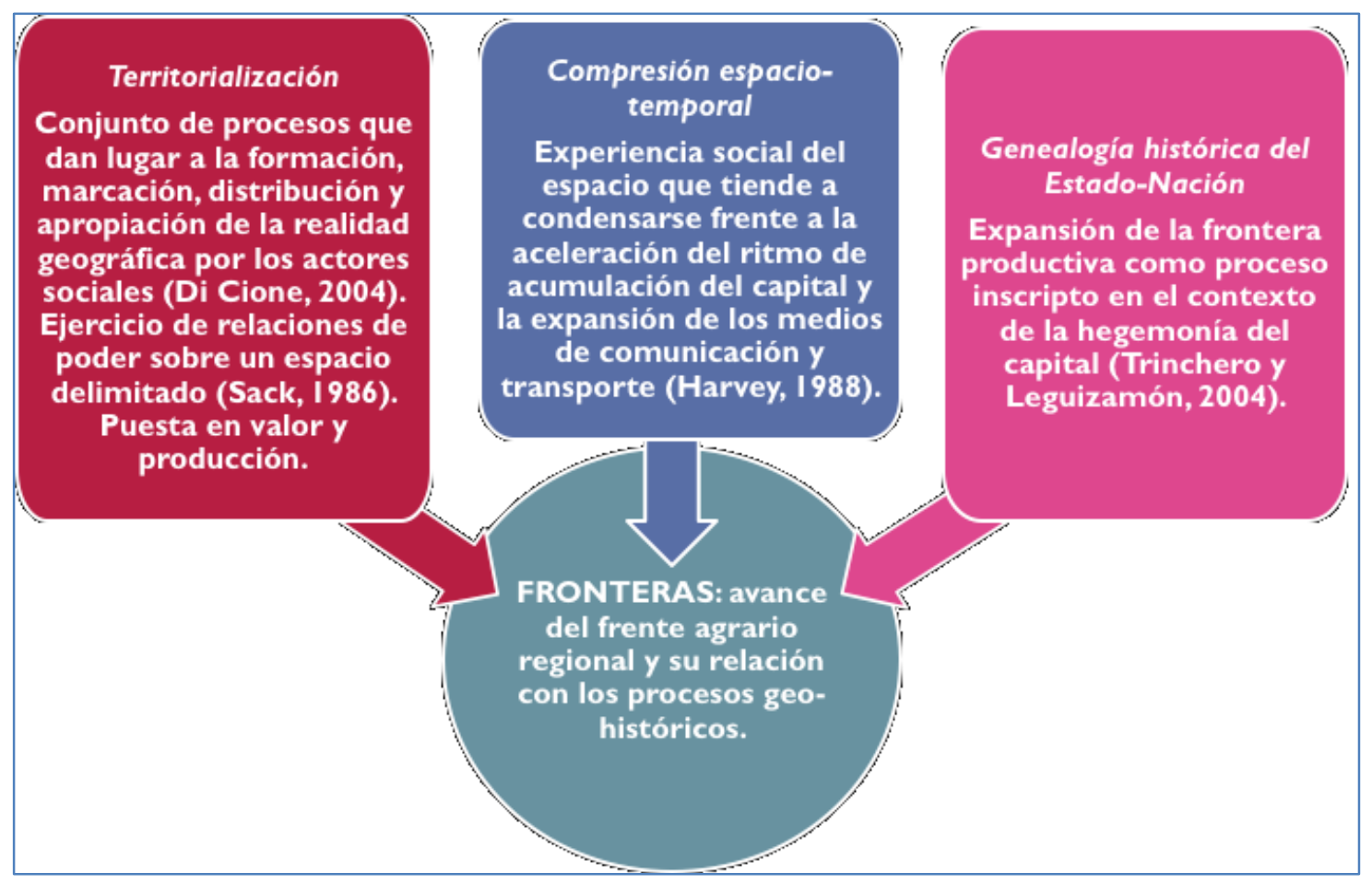

Fuente: elaboración propia en base a Harvey (1988); Sack (1986); Trinchero y

Leguizamón (2004).

En efecto, la categoría posee rasgos territoriales que no se rigen exclusivamente en función de la consolidación de las fronteras estatales, sino también gracias a la lógica de 
apropiación del espacio con cada extensión de las fronteras internas y agropecuarias. Así, el avance de las fronteras de los estados nacionales y las fronteras productivas se han combinado, reproduciendo formas espaciales particulares que se caracterizan por la generación de economías de enclave junto con la apropiación diferencial de la tierra (Figura 1).

La consolidación del Estado-Nación argentino y la expansión de la frontera agraria se superponen a finales del siglo XIX en distintas regiones, como la Patagonia y el Chaco. El territorio nacional queda delimitado desde la perspectiva del ejercicio de las relaciones de poder del aparato estatal y -de este modo- se sientan las bases para el nuevo usufructo del espacio, ahora libre de población indígena. En este sentido, podría hablarse de una acumulación originaria a escala regional. Siguiendo a Harvey (2003), cualquier espacio que pertenece a una formación social, y que se incorpora a la lógica de desarrollo capitalista, sufre transformaciones estructurales. Las mismas que Marx denominaba como parte del proceso de acumulación originaria.

\section{Frente, frontera productiva y formación social de fronteras}

La procedencia del término frontera deriva de frente, un término militar que designa la zona de contacto con una armada enemiga. Con la consolidación del Estado-Nación moderno durante los siglos XVIII y XIX, la "frontera" se convierte en sinónimo de "límite" entre los estados. De esta manera, el término adquiere un sentido principalmente geopolítico y comienza a ser asimilado a la concepción de los límites como algo dado, natural. Así, la mayoría de las fronteras entre los estados se intentan establecer sobre alguna característica física del terreno (Reitel y Zande, 2004).

Más tarde, aparecen algunas distinciones entre conceptos asociados a la frontera. Una de ellas es la diferencia entre las expresiones border y frontier. La primera refleja el límite territorial entre la jurisdicción de distintos Estados-Nación. Por su parte, el segundo remite a la articulación entre dos sistemas socio-espaciales diferentes; “donde un frente de inversiones capitalistas y estatales tiene una tendencia a expandirse sobre una zona marginal" (Gordillo y Leguizamón, 2002: 15).

Por su parte, con la categoría formación social de fronteras se intenta penetrar el proceso de constitución del Estado argentino a través de la consolidación de las fronteras políticas 
(borders) en combinación con la apropiación y valorización de los espacios vacíos en términos productivos (frontiers). En este sentido, Hugo Trinchero afirma:

"La construcción de la nacionalidad inscripta en la ocupación de espacios vacíos, construye un otro en términos de enemigo; que el énfasis puesto en los conflictos territoriales facilita la institucionalización del uso de la violencia armada; que las relaciones de producción presentes en la formación social de fronteras Chaco Central, generan mecanismos de coerción política y militar para garantizar el proceso de valorización; que la construcción de tipologías étnicas, estigmatiza los significantes de las contradicciones que en determinados momentos aparecen entre la reproducción de la vida y la reproducción del capital; que la naturalización del estigma étnico, oscurece las trayectorias sociales de los pobladores y la dinámica de los procesos de subsunción del trabajo y reproducción de la vida, al capital; y finalmente, que la construcción de un escenario de revalorización del territorio y de la fuerza de trabajo, dilata la regularización dominial de sus ocupantes criollos y aborígenes" (2000: 275-276).

Siguiendo esta línea de razonamiento, la expansión de la frontera agraria se desarrolló de manera concomitante al proceso de territorialización del Estado (entendido como el ejercicio efectivo de control sobre el territorio). Durante el proceso formativo del Estado argentino (1870-1930) - una vez controlado el espacio de las fronteras internas a finales del siglo XIX- comienza a consolidarse el aparato productivo nacional orientado a la exportación de materias primas. De esta manera, los dispositivos de control y disciplinamiento sociales en la intervención del "espacio vacío" indígena se articularon con las hipótesis de conflicto hacia las fronteras externas (Trinchero, 2007).

\section{La formación social puneña}

Para comenzar, se puede señalar la importancia toponímica que posee el término "puna" sobre la región objeto, especialmente en territorio argentino. Esencialmente, tiene un enorme contenido simbólico para la geografía interior de la Argentina. Según el discurso estatal "homogeneizador", se trata de un "espacio vacío", una geografía física despoblada; así, incluso descripciones recientes enfatizan en este tono argumentativo. De este modo, la concepción naturalista de origen decimonónico persiste en la clasificación 
actual de la Puna; y al igual que con otras regiones del país, invisibilizan a las poblaciones indígenas locales, reforzando la necesidad estatal de ejercicio de territorialidad sobre dicho espacio.

Asimismo, la invención de un imaginario geográfico regional por parte del EstadoNación (basado en los aspectos naturales del área), consolidó los dispositivos discursivos sobre este territorio, tornando a la "puna" como categoría narrativa central en el proceso de apropiación, delimitación y distribución del espacio analizado (Benedetti, 2014). Una primera aproximación puede realizarse del lado argentino, donde varias provincias comparten una porción de esta elevada planicie al este de la Cordillera de los Andes, a más de $3.500 \mathrm{msnm}$. Son estados federales de segundo nivel, que forman parte de la región Noroeste de la Argentina (NOA): las provincias de Jujuy, Salta y Catamarca. Siguiendo a Teruel:

“Al comenzar el siglo XX, la Puna argentina era una de las tantas áreas de América Latina en la que pervivían importantes sectores de economía de autosubsistencia, con distintos grados de articulación a los mercados locales y regionales. Era también una de las áreas en las que el capitalismo había provocado cambios, pero sin llegar a transformar radicalmente su característica de sociedad campesina, la que, por otra parte, le resultaba funcional" (2005: 2-3).

Parte de la reproducción anual de estas unidades campesinas se sustentaba sobre el trabajo doméstico, que durante cierto tiempo del año se emplearían como fuerza de trabajo en ingenios, minas o latifundios. Así sucedió a lo largo de la región NOA, donde predominó el cultivo de azúcar sobre los valles a partir de la instalación de gran cantidad de ingenios. De manera concomitante, el avance de otros cultivos al correr del siglo pasado (como oleaginosas, cítricos y tabaco, principalmente) incrementó la necesidad de mano de obra. La zona fronteriza con Bolivia sobre la Puna pertenece a la Provincia de Jujuy, en el extremo norte de la Argentina. Hasta principios del siglo XX se articulaba una coexistencia entre el modelo agroindustrial descripto y la economía ganadera del altiplano. Sin embargo, para el Censo Nacional de 1914 la población en la Puna jujeña sólo representaba el 16\% de la provincia (Ibídem). La principal causa de migración era la demanda de fuerza de trabajo en los valles dedicados a la agricultura. La creciente 
proletarización de la población de la Puna (otrora arrendataria) se canalizó en la agroindustria azucarera primero, y en la minería a gran escala después (Fandos, 2016).

Ahora bien, si se posiciona el análisis específicamente sobre la frontera, una vez consolidados los límites jurídicos internacionales, comienzan a ponerse en práctica dinámicas transfronterizas propias y distintivas de la formación social de fronteras. En este sentido, la integración física a través del ferrocarril y la resolución de las disputas de límites durante la década de 1920 del siglo pasado, dio el puntapié inicial para período de fronterización (Benedetti y Salizzi, 2011). El primer puesto de avanzada del Estado argentino se realizó con el emplazamiento de la aduana de La Quiaca, localidad fundada en 1883. Así, se centralizaría en un único paso fronterizo todos los flujos dispersos a lo largo de la extensa frontera en la formación social puneña. Un poco más tarde, la fundación de Villazón y la construcción del ferrocarril, vendrían a consolidar el límite binacional.

Por otra parte, pero no menos importante, era la consolidación territorial por parte de los Estados-Nación en la formación social de fronteras de la Puna. En efecto, la expansión de la red ferroviaria constituyó el principal sostén para la integración física en áreas marginales donde el sistema ferroviario de origen privado británico no encontraba rentabilidad. Para 1890, el tren llegaba a San Salvador, y más tarde conectaría con La Quiaca en la frontera con Bolivia, a partir de 1908. Según Benedetti:

"El ferrocarril fue clave para el despegue de la economía capitalista azucarera centrada en los valles subtropicales del oriente jujeño, que se transformó en el principal centro gravitacional de la provincia. De todas formas, el servicio ferroviario acompañó algunas transformaciones del eje del río Grande. Hasta entonces una de las principales actividades era la provisión de pasturas para el ganado en pie que iba hacia las minas del sur de Bolivia, además de la agricultura y la ganadería de autosubsistencia” (2015: 32).

Poco después, durante la década de 1920 se extendería a Tupiza y Uyuni, al sur de Bolivia, y así con La Paz más al norte y con el Océano Pacífico a través del puerto de Antofagasta en Chile. Dicha situación serviría para conectar los territorios puneños de tres países: Argentina, Bolivia y Chile. Así se consolidarían actividades tales como el turismo en la Quebrada de Humahuaca, y más al norte todas las relacionadas con el complejo de 
extracción minera. No sólo la explotación de boratos, plata, plomo y zinc; sino también la construcción talleres y depósitos ferroviarios, con el consecuente surgimiento de los pueblos donde se desarrollarían estos emprendimientos (Figura 2).

Figura 2. Red ferrocarriles circumpuneña.

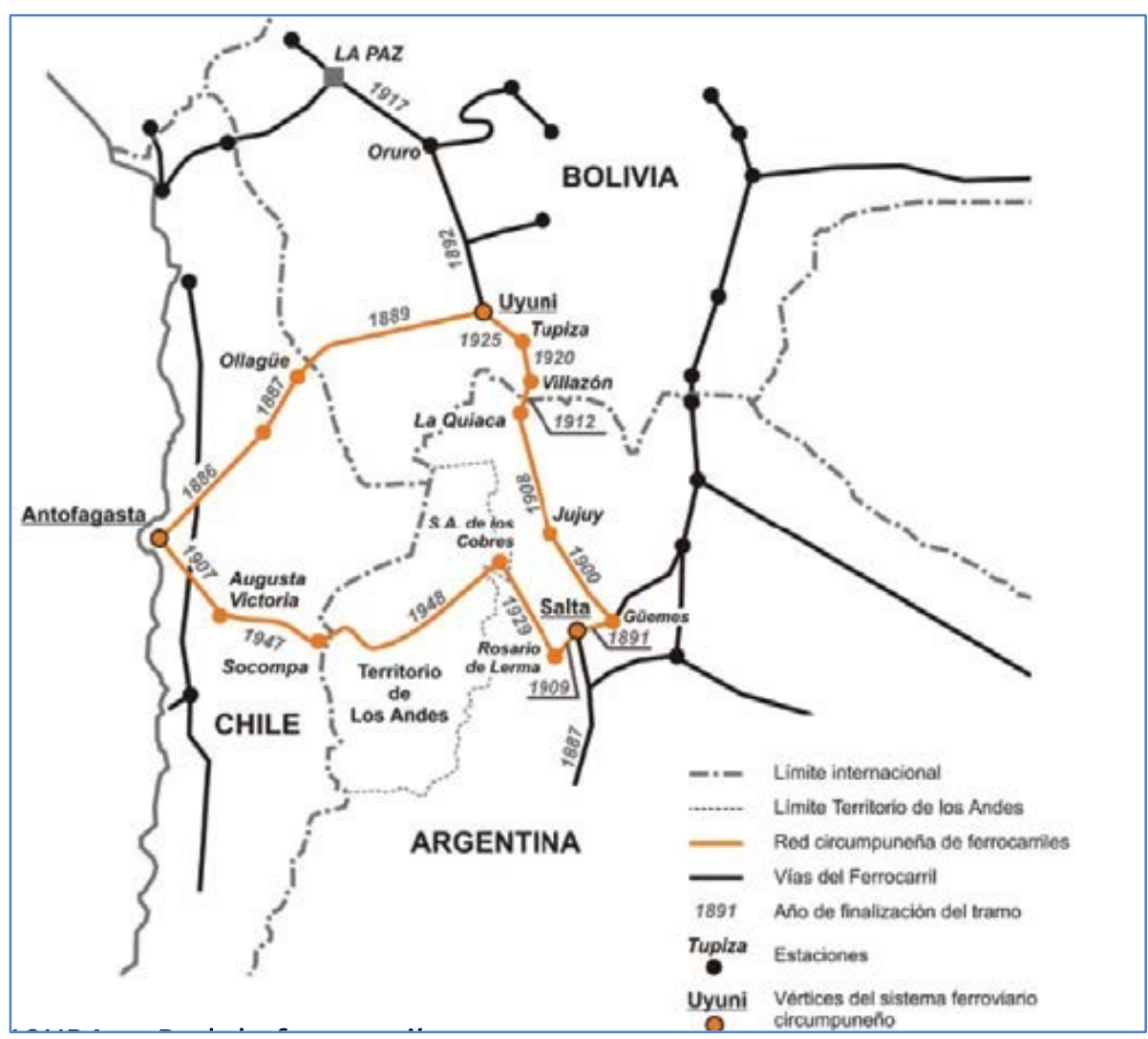

Fuente: Benedetti (2015).

Tiempo después, con el desarrollo del sistema carretero nacional, la Ruta Nacional 9 conectaría a San Salvador de Jujuy con Buenos Aires; no obstante, recién durante los años setenta alcanzaría la localidad de Humahuaca. En la década del noventa, se clausuraría definitivamente el tren, y recién en los años 2000 la Provincia de Jujuy quedaría conectada a través de rutas asfaltadas con Bolivia -RN 9- y Chile-RN52- (Ibídem).

En este punto, es interesante rescatar la noción de "extracéntrico", en contraposición al binomio centro-periferia, con la intención de reflexionar sobre la relación de estos espacios con otros centros, más allá de su vinculación con la metrópoli (Martínez, 2013; Teruel y Elbirt, 2019). De manera más dinámica, pensar las interacciones al interior de la 
región objeto también implica identificar las características del desarrollo desigual y combinado en una zona de fronteras. Si bien no es la finalidad del presente trabajo, es importante comprender la relación entre los centros regionales del norte argentino y el sur boliviano, con respecto al resto de la región; ya que estas interrelaciones superan los límites jurisdiccionales. En la actualidad, existen nexos comerciales que exceden tanto a la Puna, como a las regiones centrales de cada país, como por ejemplo entre Santa Cruz de la Sierra, en el oriente boliviano y la Provincia de Salta, más al sur de Jujuy.

A modo comparativo, con el propósito de ejemplificar y describir con datos estadísticos, cuando se hable de la región fronteriza de la Puna argentina, se tomará como unidad de análisis a la Provincia de Jujuy, territorio que presenta una enorme multiplicidad de interacciones con la República de Bolivia. En este sentido, para el Censo Nacional de 2010 un 4\% de la población total era de origen boliviano, mientras que esta nacionalidad representaba mas del 90\% de la población extranjera, situación que demuestra los fuertes lazos entre el sur y el Altiplano boliviano con el norte argentino, ya que una porción considerable de esta inmigración provenía de La Paz, Cochabamba y Potosí. Por su parte, el aglomerado San Salvador-Palpalá concentraba casi la mitad de los habitantes de la provincia, mientras que para 2018 se proyectaba una población de 750.000 habitantes; en tanto que la porción urbana alcanzaba un $70 \%$ y la población con $\mathrm{NBI}^{3}$ era de un $18,2 \%$ (DiPEC, 2013).

En materia económica, casi un $20 \%$ de la producción local se ubica en la industria manufacturera, dato que merece una breve aclaración, ya que se trata de materias primas que se transforman en productos finales o intermedios con bajo procesamiento. Las actividades de origen son la agricultura (azúcar y tabaco), la ganadería (porcina y camélida), la forestal, la minera y la acerera. Asimismo, otro $20 \%$ se ve constituido por la actividad comercial mayorista y minorista. En tercer lugar, se encuentran "educación" y "transporte-comunicaciones", con alrededor de un 10\% cada rubro; mientras que “agricultura, ganadería, pesca y silvicultura" alcanza un 9\% (Medina, 2017). El PBG

\footnotetext{
${ }^{3}$ Según el Instituto Nacional de Estadísticas y Censos (INDEC) los hogares con Necesidades Básicas Insatisfechas (NBI) son aquellos que presentan al menos una de las siguientes condiciones de privación; hacinamiento: hogares con más de tres personas por cuarto; vivienda: hogares que habitan una vivienda de tipo inconveniente; condiciones sanitarias: hogares que no tienen retrete; asistencia escolar: hogares que tienen al menos un niño en edad escolar (6 a 12 años) que no asiste a la escuela; capacidad de subsistencia: hogares que tienen cuatro o más personas por miembro ocupado, cuyo jefe no hubiese completado el tercer grado de escolaridad primaria (2010).
} 
industrial se registra en un 24\% del total (MECON, 2015). Por último, según la Encuesta Permanente de Hogares (EPH), el ingreso promedio per cápita para el tercer trimestre de 2017 era de USD 4.662 (INDEC, 2017).

En efecto, se trata de una economía de base primaria y de servicios, con escaso grado de industrialización, pese a contar con 900 emprendimientos que sostienen más de 10.000 puestos de trabajo. De este universo, seis son grandes empresas privadas que dedicadas a los siguientes rubros: producción azucarera (Ledesma y Rio Grande); minería metálica y no metálica (El Aguilar y Holcim, respectivamente); tabacalera (Cooperativa Tabacalera de Jujuy) y acerera (Aceros Zapla).

\section{El espacio del Altiplano}

En la identificación del espacio al norte de la frontera argentina, ha sido difícil conseguir información socio-territorial específica al caso; más bien bibliografía de corte histórico y arqueológico que, de todos modos, ha sido de gran utilidad. Distintos referentes académicos definen a esta porción de territorio como "Altiplano", "Altiplano central" o "Altiplano boliviano" (Nielsen, 2002; Tarragó, 1984). Asimismo, también se lo ha regionalizado de este a oeste a partir de la orografía de sus tres cadenas montañosas: oriental, central y occidental; mientras que de norte a sur se le denomina de la siguiente manera: septentrional, central y meridional.

Es interesante rescatar aquí la visión de Tarragó sobre "frontera blanda", preexistente a la constitución de los estados nacionales, colocando el eje sobre la región circumpuneña (que puede observarse en la Figura 2). Así, la autora identifica este espacio donde "al mismo tiempo que se han dado esferas de articulación, se han producido procesos diferenciales con respecto a los complejos de desarrollo altiplánicos de otras regiones" (1984: 93). Si bien remite a situaciones muy anteriores a las analizadas en el presente artículo, puede afirmarse que se trata de una región con diversos niveles de integración en diferentes procesos socio-territoriales y económicos, que perduran hasta la actualidad. En el tiempo presente, las principales actividades se basan en la minería (zinc, plata, plomo y boratos), la ganadería (bovina, caprina, ovina y llamas) y la agricultura sobre los valles más bajos (cebada, maíz, habas, trigo y papa). Las principales ciudades mineras son Potosí (nombre homónimo del departamento), Uyuni y Llallagua; la zona agrícola- 
ganadera se estructura sobre el valle del río Tupiza, mientras que la ciudad de Villazón representa el área de intercambio fronterizo con Argentina.

Para el caso boliviano, se tomará como unidad de análisis al Departamento Potosí. Según cifras oficiales, para el año 2017 el 41\% del Producto Bruto Interno (PBI) se corresponde con la actividad extractiva de minas y canteras, mientras que en segundo lugar se posicionan los servicios de la administración publica con un 17\%, seguido por la agricultura, silvicultura, caza y pesca con un 10\%; en cuarto lugar, el comercio con $8 \%$ y en quinto transporte, almacenamiento y comunicaciones alcanzando un 7\%. Mientras tanto, el PBI per cápita para 2005 se registraba en USD 553. Doce años después, para 2017 se ubicaba en USD 2.821. Este registro implica un crecimiento considerable, que se replicó a lo largo de toda Bolivia; mostrando así un desarrollo económico y social sostenido en el tiempo desde el año 2005.

Según las proyecciones demográficas, el Departamento de Potosí registra una población similar a la de la Provincia de Jujuy, que se traduce en 880.000 habitantes para el año 2018, mientras que la porción urbana representa apenas un 42\% (INE, 2018a). Más abajo, se pueden observar las cifras de NBI para el año 2012 (Figura 3). Las áreas con porcentajes más elevados representan espacios rurales, mientras que el promedio departamental asciende a 59,7\% (INE, 2018b). Si se lo compara con la Provincia de Jujuy, se trata de un promedio significativamente mayor, pese a la notable evolución de los indicadores macroeconómicos recientes.

Figura 3. Porcentaje población con necesidades básicas insatisfechas (NBI). Departamento Potosí, Bolivia. Año 2012. 


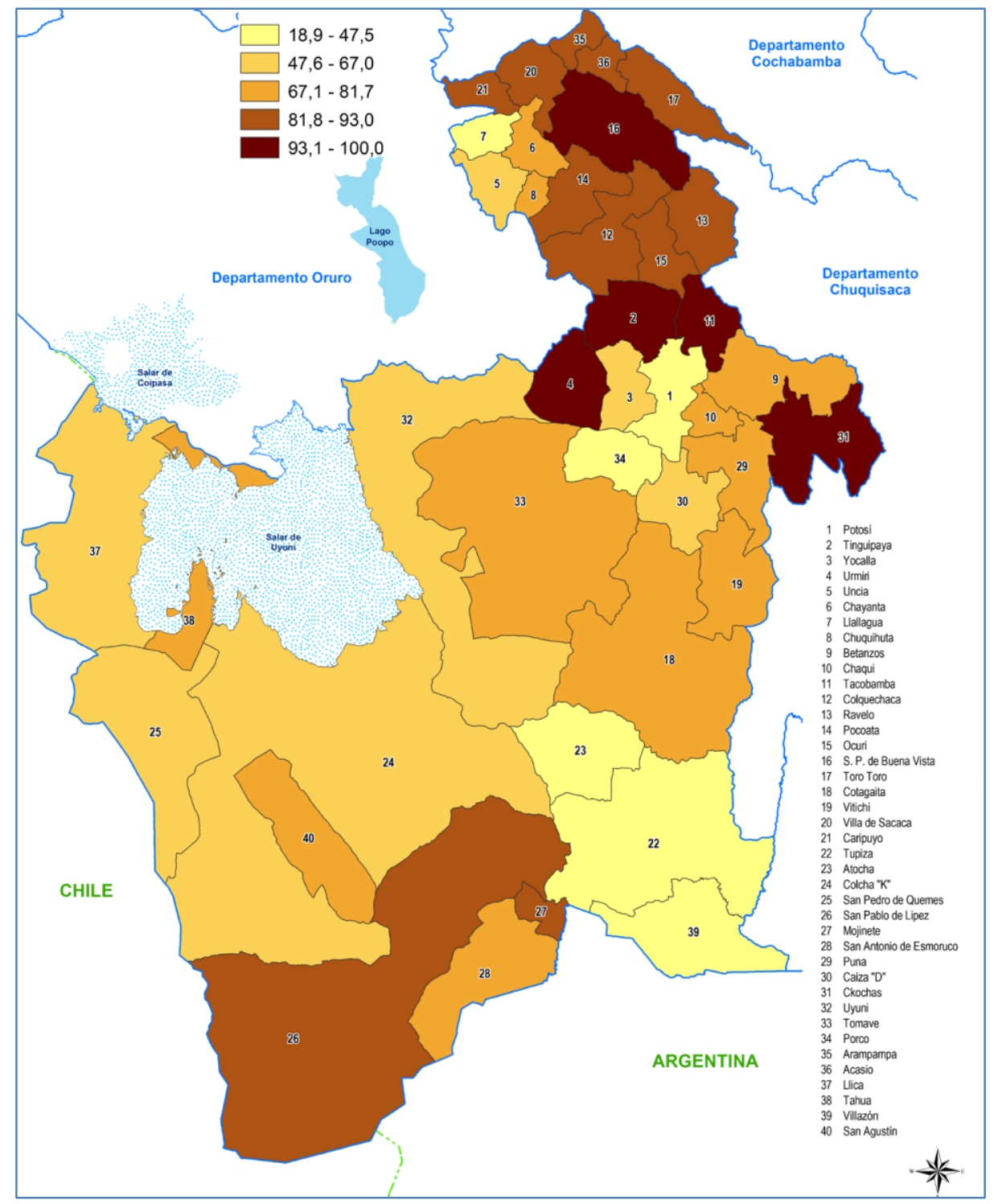

Fuente: Ibídem.

\section{Niveles recientes de integración transfronteriza}

Desde la década del ochenta, comienza un nuevo período de "tenue integración fronteriza" a nivel macro, a partir de la constitución de un mercado económico común (MERCOSUR); además de iniciativas como IIRSA (Integración de la Infraestructura Regional Suramericana) y ZICOSUR (Zona de Integración del Centro Oeste Sudamericano). A nivel meso y micro se desarrollaron los motores de la paradiplomacia, como los Comités de Frontera destinados a la resolución de cuestiones domésticas 
binacionales. Asimismo, puede destacarse que la conurbación transfronteriza aglutina a más de 90.000 habitantes repartidos entre Villazón y La Quiaca (Benedetti, 2015).

Para el caso del comercio transfronterizo, no se trata de una espacio de gran flujo comercial, ya que la integración regional se observa mucho más avanzada de este a oeste, como lo son los ejemplos de Bolivia con Chile y Perú, debido a la inclinación de gran parte de su comercio hacia el Océano Pacífico; del mismo modo, el desarrollo económico cisplatino de la República Argentina ha estado -históricamente- orientado hacia el puerto de Buenos Aires, siendo en la actualidad Brasil el principal socio regional.

Así las cosas, el espacio fronterizo de la formación social puneña no parece representar una alternativa tan destacada para el comercio internacional de la región. De manera reciente, gracias a las buenas cifras macroeconómicas que presenta el Estado Plurinacional de Bolivia, se han venido asfaltando caminos y rutas, como es el caso de la conexión Villazón-Tupiza- Potosí con el norte del país, además de la ciudad de Uyuni con el corredor sur de Tupiza y Villazón. Todo esto, ha redundado en una mejora ostensible de la conectividad, incrementando las opciones de las movilidades orientadas al intercambio y al turismo.

Otras de las cuestiones a tener en cuenta se relacionan con el pequeño comercio, los intercambios cotidianos y las relaciones parentales que se desenvuelven a lo largo de la frontera analizada. Gran parte de estas transacciones están determinadas por las variaciones en el tipo de cambio. Como se ha venido observando durante los últimos dos años, la macroeconomía argentina ha mostrado serias deficiencias en materia de déficit, especialmente en cuanto a su capacidad de respaldar la moneda local en relación a sus reservas en dólares, que históricamente se han ubicado por debajo de la demanda doméstica de la divisa extranjera.

En este sentido, la menor oferta -además de los desaguisados económicos por parte de la gestión de gobierno- han multiplicado la cotización del dólar por cuatro (desde los ARS15 de diciembre de 2015 a los ARS60 de octubre de 2019). Estas variaciones constantes, intensificadas a lo largo de los últimos veinte meses, han propiciado que los intercambios (basados en un tipo de cambio atrasado) se hayan desmoronado de manera notable. Esto puede verificarse en los comercios que se encuentran en Villazón, que observaron una reducción ostensible en la demanda, pese a comercializar productos importados a precios 
relativamente bajos en dólares (principalmente con origen en China y el Sudeste Asiático).

\section{Un apartado al final}

Brevemente, se puede señalar la complejidad que presenta la formación social de fronteras analizada, en términos sociales, económicos, administrativos y culturales; con especial énfasis en lo referido a la frontera binacional entre Argentina y Bolivia. Allí los diversos procesos de fronteras convergen de manera específica a través de los intercambios cotidianos, las relaciones de parentesco y las vicisitudes económicas propias de este espacio. Si se extiende un poco la escala, se trata de un territorio mucho más extenso que se encuentra estrechamente relacionado con la mencionada frontera. Como se describió, la formación social puneña comprende parte del espacio nacional boliviano y argentino -también chileno- aunque sólo se abordaron los dos primeros. Así, se explicitó que las diferentes dinámicas locales trascienden sus espacios nacionales de referencia.

Para indagar más adelante, se propone adicionar al espacio chileno, con el propósito de complejizar el análisis; realizando así una descripción pormenorizada sobre las actividades sociales, económicas, espaciales y culturales a lo largo y ancho de la formación social objeto. Si bien existe gran cantidad de bibliografía referente a cuestiones históricas, étnicas y arqueológicas, sería necesario ahondar respecto de las características actuales en materia económica, social y geográfica. A partir de este objetivo central, sería recomendable identificar a los actores sociales intervinientes en la "frontera", tales como productores, agentes administrativos, comunidades locales y comerciantes a cada lado del límite internacional. Representa el desafío para completar esta investigación en la formación social de fronteras de la Puna.

Por último, pero no menos importante, se pueden señalar los acontecimientos ocurridos en el Estado Plurinacional de Bolivia, que se fueron sucediendo durante la escritura del presente ensayo. Sería interesante incorporar el análisis geopolítico en trabajos venideros, con nociones tales como las etnicidades y su relación con el Estado-Nación, concepto que se aborda desde la noción de formación social de fronteras. Por su parte, la desestabilización reciente del sistema constitucional boliviano, produciendo situaciones de severa "anomía institucional", se traduce en un posible escenario de "Estado fallido" 
(Chomsky, 2007; Zapata Callejas, 2014). Dicha situación, no sólo aplicaría para Bolivia; más bien se trataría de un formato para la apropiación de los recursos naturales en toda la región, tal es el caso del norte de África y parte de Medio Oriente. Simplemente, se trata de una digresión para pensar futuras investigaciones de corte económico y geopolítico, pendientes para el área objeto.

\section{Bibliografía}

ALTHUSSER, L. Y BALIBAR, E. (2001) [1974]: Para leer El Capital. Siglo XXI Editores. México DF, México.

BENEDETTI, A. (2014): “¿Qué es la Puna? El imaginario geográfico regional en la construcción conceptual del espacio argentino (siglos XIX y XX)” en Espacialidades altoandinas. Nuevos aportes desde la Argentina. Tomo II: Interacciones con el mundo de afuera. Benedetti y Tomasi (Compiladores). Facultad de Filosofía y Letras. Buenos Aires, Argentina (pp. 5-73).

(2015): "Breve hodografía histórica de la Quebrada de Humahuaca". El Ojo del Cóndor, 6. Instituto Geográfico Nacion. Buenos Aires, Argentina (pp. 30-35).

BENEDETTI, A. Y SALIZZI, E. (2011): “Llegar, pasar, regresar a la frontera. Aproximación al sistema de movilidad argentino-boliviano". Revista Transporte y Territorio, 4. Facultad de Filosofía y Letras. Buenos Aires (pp. 148-179). Recuperado de: http://www.rtt.filo.uba.ar/RTT00409149.pdf

CHOMSKY, N. (2007): Estados Fallidos: el abuso de poder y el ataque a la democracia. Ediciones B. Barcelona, España.

DiPEC (2013): Anuario Estadístico de la Provincia de Jujuy. Dirección Provincial de Estadísticas y Censos. San Salvador de Jujuy, Argentina. 
FANDOS, C. (2016): "Niveles de Vida de los Arrendatarios de Cochinoca (Jujuy). Entre el dominio del Marquesado de Tojo y la propiedad fiscal, siglo XIX". Folia Histórica, 26. IIGHI-CONICET. Resistencia, Argentina.

GORDILLO, G. (1992): "Procesos de subsunción del trabajo al capital en el capitalismo periférico" en Antropología Económica II. Conceptos fundamentales. Trinchero (Compilador). CEAL. Buenos Aires, Argentina (pp. 45-67).

(1995): “La subordinación y sus mediaciones: dinámica cazadorarecolectora, relaciones de producción, capital comercial y Estado entre los tobas del oeste de Formosa" en Producción doméstica y capital: estudios desde la antropología económica. Trinchero (Compilador). Editorial Biblos. Buenos Aires, Argentina (pp. 105-137).

GORDILLO, G. Y LEGUIZAMÓN, J.M. (2002): El río y la frontera. Editorial Biblos. Buenos Aires, Argentina.

HARVEY, D. (1988): La condición de la Posmodernidad. Editorial Amorrortou. Buenos Aires, Argentina. (2003): El nuevo imperialismo. Editorial Akal. Buenos Aires, Argentina.

INDEC (2017): Encuesta permanente de hogares. Aglomerado San Salvador-Palpalá. Tercer trimestre de 2017. Instituto Nacional de Estadísticas y Censos. Buenos Aires, Argentina.

INE (2018a): Boletín Informativo Departamento Potosí 2018. Instituto Nacional de Estadísticas. La Paz, Bolivia.

(2018b): Anuario Estadístico 2017. Instituto Nacional de Estadísticas. La Paz, Bolivia. 
MARTÍNEz, A. (2013): “Intelectuales de provincia: entre lo local y lo periférico". Prismas, 2, (17), (pp.169-180).

MARX, K. (2001) [1857]: Introducción general a la crítica de la economía política. Siglo XXI Editores. México DF, México.

MECON (2015): Ficha Provincial Jujuy. Ministerio de Economía, Secretaría de Política Económica y Planificación del Desarrollo. Buenos Aires, Argentina.

MEDINA, F. (2017): “Estimación del Producto Bruto Geográfico de Jujuy (20042016)". Documento de Trabajo, 34. SIMEL Nodo NOA/FCE- FHyCS/UNJu. San Salvador de Jujuy, Argentina. Recuperado de: www.fce.unju.edu.ar/simel

NIELSEN, A. (2002): “Asentamientos, conflicto y cambio social en el Altiplano de Lípez (Potosí, Bolivia)". Revista española de antropología americana, 32. Universidad Complutense. Madrid, España (pp. 179-205).

REITEL, B. Y ZANDE, P. (2004): Definición de Frontera. Hypergeo. Recuperado de: http://www.hypergeo.eu/spip.php?article326

SACK, R. (1986): Human territoriality its theory and history. Cambridge University Press, Nueva York, Estados Unidos.

TARRAGÓ, M. (1984): “La historia de los pueblos circumpuneños en relación con el altiplano y los Andes Meridionales”. Estudios Atacameños, 7 (pp. 93-104). Recuperado de: https://doi.org/10.22199/S07181043.1984.0007.00010

TERUEL, A. (2005): "Estructuras agrarias comparadas: la Puna argentina y el sur boliviano a comienzos del siglo XX". Mundo Agrario, 6, (11). Recuperado de: http://www.memoria.fahce.unlp.edu.ar/art_revistas/pr.545/pr.545.pdf 
TERUEL, A. Y ELBIRT, A. (2019): “La frontera argentino-boliviana. Una mirada desde el servicio diplomático argentino". Actas del X Congreso Internacional de la Asociación de Estudios Bolivianos. Sucre, Bolivia.

trinchero, H. (2000): Los dominios del demonio. Editorial Eudeba, Buenos Aires, Argentina.

(2007): Aromas de lo exótico (retornos del objeto): para una crítica del objeto antropológico y sus modos de reproducción. Editorial SB. Buenos Aires, Argentina.

TRINCHERO, H., BELLI, E. Y SLAVUTSKY, R. (2004): La cuenca del río Bermejo. Una formación social de fronteras. Editorial Reunir. Buenos Aires, Argentina.

TRINCHERO, H. Y LEGUIZAMÓN, J.M. (1995): "Hidrocarburos, dinámica del capital y cuestión social en el norte argentino" en La cuenca del río Bermejo. Una formación social de fronteras. Trinchero, Belli y Slavutsky (Compiladores). Editorial Reunir. Buenos Aires, Argentina.

TRINCHERO, H., PICCININI, D. Y GORDILLO, G. (1992). Capitalismo y grupos indígenas en el Chaco Centro-Occidental (Salta y Formosa/2). CEAL. Buenos Aires, Argentina.

ZAPATA CALLEJAS, J. (2014). "La teoría del Estado fallido: entre aproximaciones y disensos". Revista de Relaciones Internacionales, Estrategia y Seguridad, 1, (9). Universidad Militar Nueva Granada. Bogotá, Colombia, (pp. 87-110). Recuperado de: https://www.redalyc.org/articulo.oa?id=927/92731211004 